\title{
Topological Junctions from Crossed Andreev Reflection in the Quantum Hall Regime
}

DOI:

10.1103/PhysRevLett.120.116801

\section{Document Version}

Final published version

Link to publication record in Manchester Research Explorer

\section{Citation for published version (APA):}

Finocchiaro, F., Guinea, F., \& San-Jose, P. (2018). Topological Junctions from Crossed Andreev Reflection in the Quantum Hall Regime. Physical Review Letters, 120(11), [116801].

https://doi.org/10.1103/PhysRevLett.120.116801

\section{Published in:}

Physical Review Letters

\section{Citing this paper}

Please note that where the full-text provided on Manchester Research Explorer is the Author Accepted Manuscript or Proof version this may differ from the final Published version. If citing, it is advised that you check and use the publisher's definitive version.

\section{General rights}

Copyright and moral rights for the publications made accessible in the Research Explorer are retained by the authors and/or other copyright owners and it is a condition of accessing publications that users recognise and abide by the legal requirements associated with these rights.

\section{Takedown policy}

If you believe that this document breaches copyright please refer to the University of Manchester's Takedown Procedures [http://man.ac.uk/04Y6Bo] or contact uml.scholarlycommunications@manchester.ac.uk providing relevant details, so we can investigate your claim.

\section{OPEN ACCESS}




\title{
Topological $\pi$ Junctions from Crossed Andreev Reflection in the Quantum Hall Regime
}

\author{
F. Finocchiaro, ${ }^{1,2}$ F. Guinea, ${ }^{2,3}$ and P. San-Jose ${ }^{1}$ \\ ${ }^{1}$ Materials Science Factory, ICMM-CSIC, Sor Juana Ines de La Cruz 3, 28049 Madrid, Spain \\ ${ }^{2}$ IMDEA Nanociencia, Calle de Faraday 9, 28049 Madrid, Spain \\ ${ }^{3}$ Department of Physics and Astronomy, University of Manchester, Manchester M13 9PL, United Kingdom
}

(Received 26 September 2017; published 15 March 2018)

\begin{abstract}
We consider a two-dimensional electron gas (2DEG) in the quantum Hall regime in the presence of a Zeeman field, with the Fermi level tuned to a filling factor of $\nu=1$. We show that, in the presence of spinorbit coupling, contacting the 2DEG with a narrow strip of an $s$-wave superconductor produces a topological superconducting gap along the contact as a result of crossed Andreev reflection (CAR) processes across the strip. The sign of the topological gap, controlled by the CAR amplitude, depends periodically on the Fermi wavelength and strip width and can be externally tuned. An interface between two halves of a long strip with topological gaps of opposite sign implements a robust $\pi$ junction, hosting a pair of Majorana zero modes that do not split despite their overlap. We show that such a configuration can be exploited to perform protected non-Abelian tunnel-braid operations without any fine tuning.
\end{abstract}

DOI: 10.1103/PhysRevLett.120.116801

During the last decade, we have witnessed a surge in both theoretical and experimental progress towards the realization of Majorana-based quantum computation [1-13]. Majorana zero modes (MZMs) are zero-energy bound quasiparticles of topological origin that are their own self-adjoint and obey non-Abelian anyon statistics. As a result, the adiabatic exchange (or "braiding") of a pair of MZMs rotates the wave function of the degenerate ground state in a noncommutative fashion [14-19]. Such a process or its generalizations [20-27] can be viewed as a coherent manipulation of qubit states realized by pairs of MZMs. The interest in Majoranabased topological quantum computation stems from the fact that, as a result of the nonlocality of the MZMs, local sources of noise do not affect the fidelity of the braiding operation, nor do they induce decoherence of the ground-state manifold. This property has inspired implementations of fault-tolerant computation schemes able in principle to beat decoherence at the hardware level [1].

The fundamental ingredient needed to create MZMs is topological superconductivity-either intrinsic, like in $p$-wave superconductors [28,29], or artificially designed, like in proximitized superconducting wires with strong spinorbit coupling (SOC) in an external magnetic field [30-32]. More recently, two-dimensional electron gases (2DEGs) with induced superconductivity are being actively investigated as platforms for topological superconductivity $[12,13$, 33-41]. In addition to the increased freedom afforded by the planar geometry, these systems allow for the formation of a new type of topological quasi-one-dimensional (1D) system, confined on both sides by two different superconductors with a phase difference $\pi$. For transparent enough contacts, such $\pi$ junctions can greatly reduce the magnetic fields required for MZMs to emerge [38,39].
In this Letter, we show that planar junctions allow for yet another implementation of 1D topological superconductivity, with a geometry dual to the above. It is achieved by contacting a long and narrow strip of a conventional superconductor with a 2DEG in the quantum Hall $(\mathrm{QH})$ regime at filling factor $\nu=1$. The proximitized region acquires a superconducting gap $\Delta$, and as a result develops gapless $\mathrm{QH}$ edge states along each side. Due to local Andreev reflection (LAR) processes, these edge states are a mixture of electrons and holes [42,43]; see Fig. 1. Assuming that spin-orbit coupling (SOC) is present in the system, and that the strip width is comparable with or smaller than the superconducting coherence length, the $\mathrm{QH}$
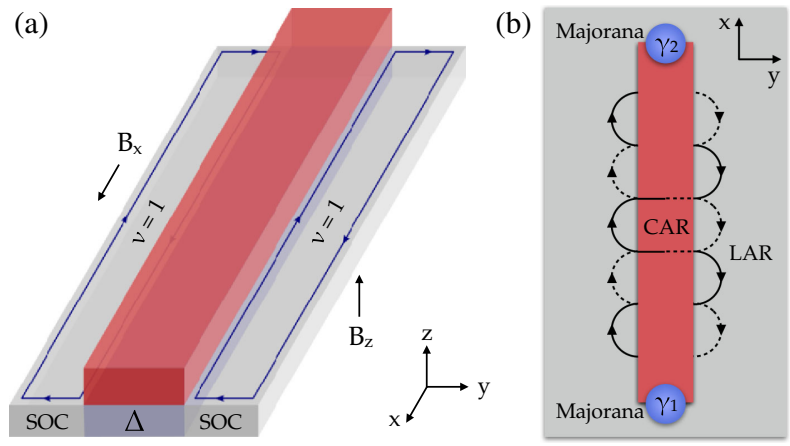

FIG. 1. (a) A $2 \mathrm{DEG}$ with strong SOC and in the $\nu=1$ state of the $\mathrm{QH}$ phase is proximitized along a narrow strip with an $s$-wave superconductor. (b) Sketch of the crossed Andreev reflection (CAR) and local Andreev reflection (LAR) processes occurring across and along the proximitized region, respectively. Full (dashed) lines represent electrons (holes). CAR processes induce a topological gap in the edge-state Majorana zero modes at the ends of the strip. 
edge states may become Cooper paired through additional crossed Andreev reflection (CAR) processes [44-48] across the strip. We show that a topologically nontrivial superconducting gap $\Delta^{*}$ then opens in the edge-state dispersion, and MZMs emerge at either end of the strip. This possibility was suggested by Lee et al. in Ref. [48], where the requisite CAR processes were experimentally demonstrated in the case of graphene, although they concentrated on the $\nu=2$ regime and not on the $\nu=1$ condition required for the formation of MZMs.

Here we theoretically investigate the conditions for CAR-induced topological superconductivity at $\nu=1$ [49]. (Related approaches have been explored in fractionalized QH systems supporting parafermions [50-52].) We find that both the magnitude and, more importantly, the sign of the topological gap depend on the amplitude of the CAR processes. As a result, the sign of $\Delta^{*}$ can be controlled by adjusting the width of the strip and/or the electronic density of the proximitized region, which in turn determine the CAR amplitude. Reeg et al. anticipated such a possibility while studying a related system of two parallel nanowires coupled through a superconductor [53]. We show that this effect may be used to induce a sign change $\Delta^{*} \rightarrow-\Delta^{*}$ along the strip by, e.g., electrostatic gating. This situation corresponds to a one-dimensional topological $\pi$ junction along the strip which is host to two degenerate MZMs that do not hybridize despite their spatial overlap $[51,54,55]$. Since the original induced $\Delta$ does not change sign (only the edge-state gap $\Delta^{*}$ does), no external fine tuning is required to maintain the $\pi$ phase difference, and the MZMs remain protected at zero energy. As we will show, this allows for a powerful generalization of tunnelbraiding strategies (originally proposed by Flensberg [56]) on the two MZMs in the junction, without the need to carefully control external parameters in the process.

Consider a normal $(N)$ 2DEG with a proximitized superconducting strip $(S)$ of width $W_{S}$ along the $x$ direction; see Fig. 1(a). The $N$ region is in the $\mathrm{QH}$ regime and is subject to a Zeeman field along $x$, allowing the electron density to be tuned to an odd filling factor $\nu=1$. (Other mechanisms, such as interaction-induced spin instabilities, may play the role of the Zeeman field in some systems $[57,58]$.) The $S$ region has uniform superconducting pairing $\Delta$ induced by proximity to the parent superconductor. We also assume that SOC is present in the system, either in the $N$ region or in the $S$ region (e.g., inherited from a superconductor made of heavy elements, such as $\mathrm{NbN}$ or NbTiN). The electronic structure of this system, obtained using a tight binding approximation on a square lattice (see the Supplemental Material [59] for details), is studied in the following.

Since the $N$ region is in the $\nu=1 \mathrm{QH}$ regime and the $S$ strip is trivially gapped, each of the two NS interfaces hosts a single spin-polarized edge state. These states travel in opposite directions at opposite interfaces [see Fig. 1(a)].
Local Andreev reflections at each interface transform the edge states into coherent superpositions of electrons and holes $[29,42,43,63,64]$, but they do not open a gap because of the chiral nature of the carriers. However, in our geometry with two parallel $N S$ interfaces at either side of the strip, another type of Andreev reflection process can take place, wherein an electron on one interface is scattered as a hole into the other interface. This crossed Andreev reflection process has a significant amplitude only for strips narrower than the coherence length $\xi \approx \hbar v_{F} / \Delta$. Unlike local Andreev reflection, CAR processes may open a superconducting gap $\Delta^{*}$ in the presence of SOC, since electron and hole edge states at opposite interfaces propagate in opposite directions at the same wave vector. The role of the SOC is to cant the spin away from the Zeeman field in opposite directions in the two edge states, so that they can pair to form a spin singlet. At $\nu=1$, the gap resulting from CAR is topological, as can be seen by a direct mapping of the two spin-canted edge states plus pairing into an Oreg-Lutchyn model [30,31] (see Eq. (B4) in the Supplemental Material]. Figure 2(a) shows the gapped band structure of an infinite strip with significant CAR processes (left, $W_{S} \simeq \xi$ ) and the gapless case without CAR (right, $W_{S} \gg \xi$ ). The topological nature of $\Delta^{*}$ manifests in the appearance of MZMs when the strip is terminated inside the 2DEG [Fig. 2(d)].

The value of the topological gap $\Delta^{*}$ is entirely determined by the CAR amplitude, which in turn depends on the strip width $W_{S}$, the Fermi wavelength $\lambda_{F}$, and the singlet amplitude governed by the proximity gap $\Delta$ and the SOC strength $\alpha$. We have performed tight binding simulations which show, specifically, that $\Delta^{*}$ is a real periodic function of the $W_{S} / \lambda_{F}$ with alternating sign; see Fig. 2(b). This behavior is confirmed by an analytical calculation in terms of Green's functions, which yields

$$
\Delta^{*} \approx \frac{4 \pi^{2} t^{\prime 2} a^{3}}{W_{S} \tilde{\lambda}_{F}^{2} \tilde{\mu}} \times \operatorname{Im}(z \csc z) \sin \theta,
$$

where $\theta$ is the spin canting angle due to spin-orbit coupling, $\tilde{\lambda}_{F}=2 \pi / \sqrt{2 m \tilde{\mu}}, \tilde{\mu}=\mu-k_{F}^{2} / 2 m, \mu$ is the strip Fermi energy, $k_{F}$ is the edge-state Fermi wave vector, and $z=$ $2 \pi \sqrt{1+i \Delta / \tilde{\mu}} \times W_{S} / \tilde{\lambda}_{F}$ (see the Supplemental Material [59] for details). This formalizes the central finding of our work. The sign of $\Delta^{*}$ follows the change in the number of normal modes in the strip, given by $\left\lfloor 2 W_{S} / \tilde{\lambda}_{F}\right\rfloor$. It is therefore likely to be realistically tuneable with electrostatic gating of the strip region that may modify both its effective width $W_{S}$ and its electronic density, or by adjusting the width lithographically [53].

The possibility of changing the sign of the topological gap along the strip opens a new opportunity for the generation of MZMs. A long strip with a uniform induced gap $\Delta$ but edge-state gaps of opposite sign in its two halves $\left(\Delta_{1}^{*} \Delta_{2}^{*}<0\right)$ forms a topological $\pi$ junction, similar to a 

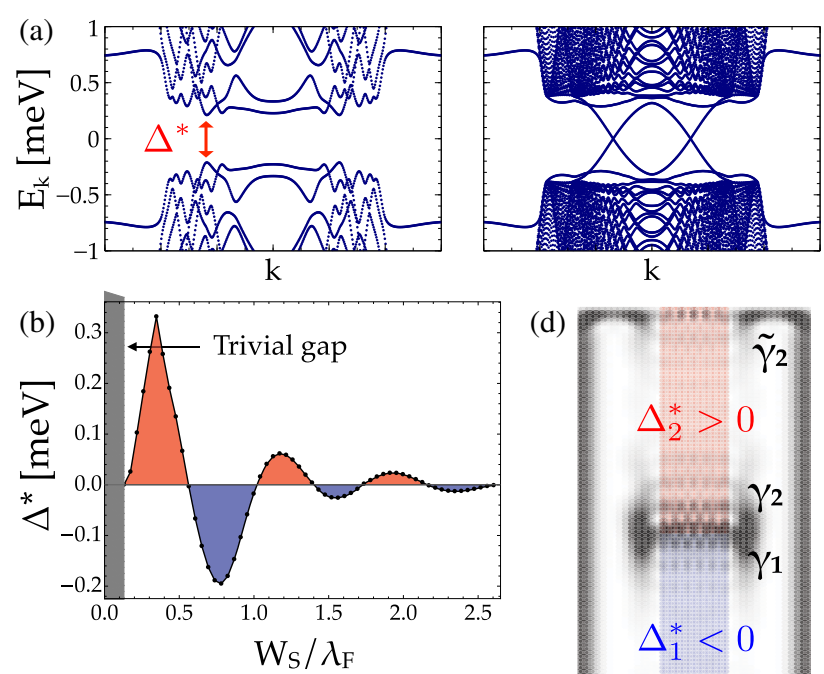

$\mathrm{k}$

(d)

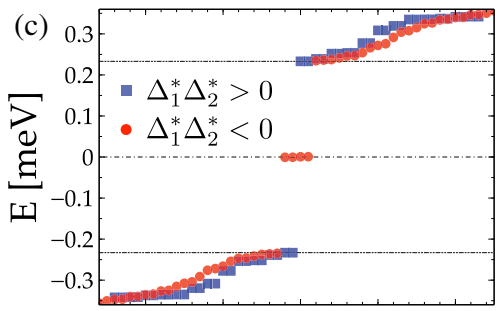

Eigenvalue

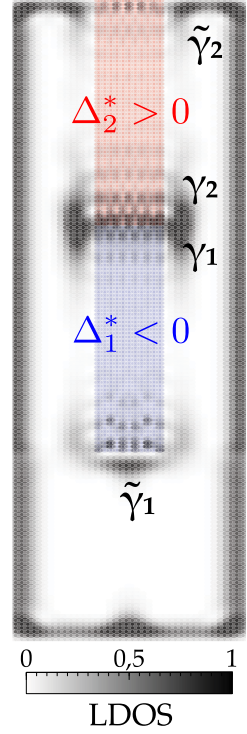

FIG. 2. (a) Spectrum of the system with periodic boundary conditions (PBCs) along both directions for widths of the central strip such that CAR is present (left, $W_{S}=300 \mathrm{~nm}$ ) and absent (right, $W_{S}=2 \mu \mathrm{m}$ ). (b) Behavior of the topological gap $\Delta^{*}$ as a function of $W_{S} / \lambda_{F}$, for $\mu / \Delta=1.95$. The grey region corresponds to the opening of a trivial gap due to the direct overlap of the $\mathrm{QH}$ edge states. (c) Lowest eigenvalues in a system with PBC and two gaps $\Delta_{1,2}^{*}$ along the strip, either of equal (blue) or of opposite sign (red). Two pairs of MZMs appear in the latter case (one pair at each of the two junctions, required in the case of PBC). (d) LDOS associated with the zero-energy eigenvalues, calculated for a system with open boundaries. The strip is such that it terminates on one end within the 2DEG and on the other at the sample edge. One Majorana mode $\left(\tilde{\gamma}_{1}\right)$ is therefore localized at one end, and the other $\left(\tilde{\gamma}_{2}\right)$ delocalizes in the $\mathrm{QH}$ edge states. The gap $\Delta^{*}$ changes sign along the strip length so that two additional nonhybridizing localized MZMs $\gamma_{1}$ and $\gamma_{2}$ appear at the boundary. See the Supplemental Material [59] for the parameters used.

topological Josephson junction tuned to phase difference $\phi=\pi$. Such a system then develops two MZMs localized at the junction [see Figs. 2(c) and 2(d)] that stay at zero energy despite their spatial overlap as long as the phase difference across the junction remains $\pi$. The $\pi$ phase difference between the two halves of the strip is robust. Since $\Delta^{*}$ on both sides is finite and real, its sign does not depend on perturbations. The CAR $\pi$ junction is furthermore stabilized by the phase rigidity of the strip order parameter $\Delta$ [59]. Unlike in $\phi=\pi$ Josephson junctions, it does not require fine tuning of any external parameter such as the flux across the superconducting circuit or the strip

parameters. As a result, CAR-induced topological superconductivity enables the creation of MZMs that remain decoupled regardless of their overlap. This offers great advantages in the context of coherent Majorana qubit manipulation and braiding, as outlined in the following section.

We now present a possible application of the CAR $\pi$ junction to the challenge of non-Abelian Majorana braiding. Plenty of proposals for the demonstration of the nonAbelian statistics of Majorana excitations have been presented, which hinge on the physical exchange (or braiding) in real space of pairs of Majorana modes $[14,15,17,18,32]$. Some other approaches, however, rest upon schemes that involve rotation of the wave function without the need for actual MZMs to move spatially [16,19-24,56]. Among these, it has been suggested [56] that adiabatic tunnel processes of single electrons from a quantum dot into pairs of Majorana zero modes can result in arbitrary non-Abelian rotations of the ground-state manifold. These so-called tunnel-braid operations are extremely versatile, as they allow a universal set of single-qubit gates, in contrast to braiding that only allows a limited set of operations. Unfortunately, tunnel braiding has the drawback of requiring a precise, typically finetuned, phase difference of $\pi$ between the MZMs involved throughout the operation. If the phase deviates from this value, the result of the operation becomes time dependent and is no longer protected against decoherence.

The robustness and lack of fine tuning of CAR $\pi$ junctions promises to overcome this problem. In Fig. 3(a), we present a possible geometry to implement a CAR-protected tunnelbraiding scheme. We deposit two narrow superconducting strips on a $\nu=1$ 2DEG such that two independent CAR-induced topological gaps $\Delta_{1}^{*}$ and $\Delta_{2}^{*}$ open on each. One end of each strip terminates inside the 2DEG, so that the corresponding MZMs $\gamma_{1,2}$ lie within a finite distance of each other. The MZMs $\tilde{\gamma}_{1,2}$ on the far end of the strips are assumed to be sufficiently far from the junction so as to become decoupled from $\gamma_{1,2}$. We control the Fermi level of the two strips, $\mu_{1}$ and $\mu_{2}$, by means of two independent gates, in order to tune the magnitude and sign of the topological gaps $\Delta_{1,2}^{*}$.

The two "inner" MZMs $\gamma_{1}$ and $\gamma_{2}$ are then coupled to a quantum dot through two tunnel barriers that may be tuned externally. The tunneling couplings $t_{1,2}$ control the specific non-Abelian operation to perform. The dot is in the Coulomb blockade regime, with occupation $N$. We adiabatically tune the dot level $\varepsilon_{D}$ across an $N \rightarrow N-1$ transition between two adjacent Coulomb valleys. This transfers a single electron to the composite state of the two Majorana modes. Figure 3(b) shows the evolution of the low-energy single-particle Bogoliubov spectrum of the full dot-2DEG-strip system across this process, with dashed lines corresponding to mostly dot states, and solid lines to MZMs states in the strip. The two cases with equal (blue, $\phi=0$ ) and opposite (red, $\phi=\pi$ ) signs for $\Delta_{1,2}^{*}$ show 
markedly different structures. The conventional $\phi=0$ case splits the MZMs away from zero close to the $N \rightarrow N-1$ transition, as they become resonantly coupled via the dot state [65]. Such an operation is not protected against noise, and its result depends on timing. In contrast, the $\phi=\pi$ case shows MZMs that remain exactly at zero energy throughout the operation, as their hybridization across the dot is forbidden by the opposite sign of $\Delta_{1,2}^{*}$. The state after emptying the dot is then independent of timing and insensitive to noise in $\varepsilon_{D}$. As shown by Flensberg [56], the transformation $P$ within the degenerate ground-state manifold associated with this process is a rotation by an angle $\pi$ around an axis in the $x y$ plane, controlled by the tunnel couplings $t_{1,2}$. If the couplings are then changed to $t_{1,2}^{\prime}$, and the reverse adiabatic transition $N-1 \rightarrow N$ on the dot is performed, the composite operation $P^{\prime} P$ rotates the quantum state of the Majorana modes by an arbitrary angle around the $z$ axis. In comparison, braiding two MZMs can only rotate the wave function about the $z$ axis by an angle of $\pi / 2$.

As no fine tuning is required to maintain the $\phi=\pi$ condition in the CAR $\pi$ junction, the tunnel-braiding process should enjoy similar topological protection as a standard spatial braiding. In Fig. 3(c), we show the MZM splitting across a resonant dot as we vary the Fermi energy under one of the strips while the other is kept fixed. As expected, we find alternating $\phi=0$ (red) and $\phi=\pi$ (blue) regions, in which the MZM splitting is finite and zero, respectively. The width in parameter space of the $\phi=\pi$ regions with MZMs pinned to zero is finite, unlike in topological Josephson junctions.

In essence, we have presented here a scheme towards one-dimensional topological superconductivity that extends previous approaches that are based on the proximity effect-i.e., local Andreev reflections-of spinless helical electronic phases coupled to superconductors. While such approaches indeed produce a topological order parameter, its phase is fixed by the parent superconductor. In contrast, crossed Andreev reflections, relevant in geometries as those discussed here, also produces a topological order parameter, but its sign may be either the same as or opposite to that of the parent, depending on the CAR amplitude itself. Controlling the sign of the topological gap in a stable way has many ramifications. We have shown how it may be exploited to produce stable, self-tuned $\pi$ junctions, wherein sizeable Majorana overlaps, which are problematic in more conventional Majorana devices, are no longer a concern, at least for pairs of MZMs at the junction. As a result, parametric braiding of Majoranas through, e.g, tunnel-braiding schemes, becomes significantly more realistic. The specific implementation of the CAR-induced topological gap described here is just one conceptually simple possibility, but it is not unique. Other phases, such as quantum anomalous Hall states, could also exhibit the requisite $\nu=1$ spin-singlet states. The temperature requirements for using our protocol are limited by both the
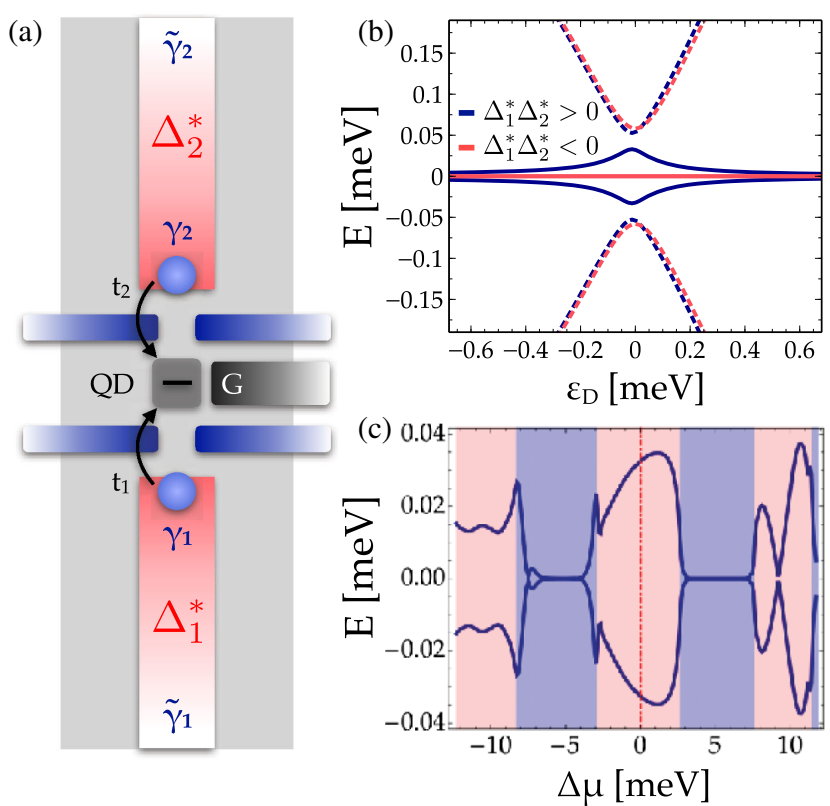

FIG. 3. (a) Sketch of a tunnel-braiding setup, with the two inner MZMs $\gamma_{1}$ and $\gamma_{2}$ from strips 1 and 2 coupled to a dot (QD) in the Coulomb blockade regime through tunnel barriers. The dot occupancy is controlled by a gate $(\mathrm{G})$, which shifts the dot level $\varepsilon_{D}$. (b) Comparison of the low-energy spectra of the composite system for gaps $\Delta_{1,2}^{*}$ in the two strips of equal (blue) and opposite (red) sign. The dotted and solid lines correspond predominantly to dot and Majorana states, respectively. (c) Energy of the MZMs as they hybridize through the dot as a function of the changing chemical potential between the left and right strips $\Delta \mu=\mu_{1}-\mu_{2}$, for $\mu_{2}$ and $\varepsilon_{D}$ fixed. The blue (dark) regions indicate phases where $\Delta_{1,2}^{*}$ have opposite sign, and $\gamma_{1,2}$ do not hybridize through the dot. See the Supplemental Material [59] for the parameters used.

Zeeman splitting and $\Delta^{*}$, which gives a conservative estimate between $0.1 \mathrm{~K}$ and $1 \mathrm{~K}$, well within reach of current experiments on this type of system. CAR-induced topological superconductivity is thus proposed as a promising road forward towards the next landmark in the field, the realization of protected non-Abelian operations in the lab.

We are grateful to L. Chirolli, E. Prada, C. Reeg, J. Klinovaja, and D. Loss for fruitful discussions. F. F. and F. G. acknowledge the financial support by MarieCurie-ITN Grant No. 607904-SPINOGRAPH. F. F. and P. S.-J. acknowledge financial support from the Spanish Ministry of Economy and Competitiveness through Grant No. FIS2015-65706-P (MINECO/FEDER).

[1] J. Alicea, Rep. Prog. Phys. 75, 076501 (2012).

[2] M. T. Deng, C. L. Yu, G. Y. Huang, M. Larsson, P. Caroff, and H. Q. Xu, Nano Lett. 12, 6414 (2012).

[3] J. R. Williams, A. J. Bestwick, P. Gallagher, S. S. Hong, Y. Cui, A. S. Bleich, J. G. Analytis, I. R. Fisher, and D. Goldhaber-Gordon, Phys. Rev. Lett. 109, 056803 (2012). 
[4] V. Mourik, K. Zuo, S. M. Frolov, S. R. Plissard, E. P. A. M. Bakkers, and L.P. Kouwenhoven, Science 336, 1003 (2012).

[5] L. P. Rokhinson, X. Liu, and J. K. Furdyna, Nat. Phys. 8, 795 (2012).

[6] A. Das, Y. Ronen, Y. Most, Y. Oreg, M. Heiblum, and H. Shtrikman, Nat. Phys. 8, 887 (2012).

[7] A. D. K. Finck, D. J. Van Harlingen, P. K. Mohseni, K. Jung, and X. Li, Phys. Rev. Lett. 110, 126406 (2013).

[8] S. Nadj-Perge, I. K. Drozdov, J. Li, H. Chen, S. Jeon, J. Seo, A. H. MacDonald, B. A. Bernevig, and A. Yazdani, Science 346, 602 (2014).

[9] M. Ruby, F. Pientka, Y. Peng, F. von Oppen, B. W. Heinrich, and K. J. Franke, Phys. Rev. Lett. 115, 197204 (2015).

[10] S. M. Albrecht, A. P. Higginbotham, M. Madsen, F. Kuemmeth, T. S. Jespersen, J. Nygård, P. Krogstrup, and C. M. Marcus, Nature (London) 531, 206 (2016).

[11] M. T. Deng, S. Vaitiekenas, E. B. Hansen, J. Danon, M. Leijnse, K. Flensberg, J. Nygård, P. Krogstrup, and C. M. Marcus, Science 354, 1557 (2016).

[12] H. J. Suominen, J. Danon, M. Kjaergaard, K. Flensberg, J. Shabani, C. J. Palmstrøm, F. Nichele, and C. M. Marcus, Phys. Rev. B 95, 035307 (2017).

[13] H. J. Suominen, M. Kjaergaard, A. R. Hamilton, J. Shabani, C. J. Palmstrøm, C. M. Marcus, and F. Nichele, Phys. Rev. Lett. 119, 176805 (2017).

[14] D. A. Ivanov, Phys. Rev. Lett. 86, 268 (2001).

[15] J. Alicea, Y. Oreg, G. Refael, F. von Oppen, and M. P. A. Fisher, Nat. Phys. 7, 412 (2011).

[16] D. J. Clarke, J. D. Sau, and S. Tewari, Phys. Rev. B 84, 035120 (2011).

[17] J. D. Sau, D. J. Clarke, and S. Tewari, Phys. Rev. B 84, 094505 (2011).

[18] B. I. Halperin, Y. Oreg, A. Stern, G. Refael, J. Alicea, and F. von Oppen, Phys. Rev. B 85, 144501 (2012).

[19] T. Hyart, B. van Heck, I. C. Fulga, M. Burrello, A. R. Akhmerov, and C. W. J. Beenakker, Phys. Rev. B 88, 035121 (2013).

[20] P. Bonderson, M. Freedman, and C. Nayak, Phys. Rev. Lett. 101, 010501 (2008).

[21] B. van Heck, A. R. Akhmerov, F. Hassler, M. Burrello, and C. W. J. Beenakker, New J. Phys. 14, 035019 (2012).

[22] P. Bonderson, Phys. Rev. B 87, 035113 (2013).

[23] S. Vijay and L. Fu, Phys. Rev. B 94, 235446 (2016).

[24] T. Karzig, C. Knapp, R. M. Lutchyn, P. Bonderson, M. B. Hastings, C. Nayak, J. Alicea, K. Flensberg, S. Plugge, Y. Oreg, C. M. Marcus, and M. H. Freedman, Phys. Rev. B 95, 235305 (2017).

[25] K. Gharavi, D. Hoving, and J. Baugh, Phys. Rev. B 94, 155417 (2016).

[26] S. Hoffman, C. Schrade, J. Klinovaja, and D. Loss, Phys. Rev. B 94, 045316 (2016).

[27] D. Aasen, M. Hell, R. V. Mishmash, A. Higginbotham, J. Danon, M. Leijnse, T. S. Jespersen, J. A. Folk, C. M. Marcus, K. Flensberg, and J. Alicea, Phys. Rev. X 6, 031016 (2016).

[28] A. Y. Kitaev, Phys. Usp. 44, 131 (2001).

[29] Q. L. He, L. Pan, A. L. Stern, E. C. Burks, X. Che, G. Yin, J. Wang, B. Lian, Q. Zhou, E. S. Choi, K. Murata, X. Kou,
Z. Chen, T. Nie, Q. Shao, Y. Fan, S.-C. Zhang, K. Liu, J. Xia, and K. L. Wang, Science 357, 294 (2017).

[30] Y. Oreg, G. Refael, and F. von Oppen, Phys. Rev. Lett. 105, 177002 (2010).

[31] R. M. Lutchyn, J. D. Sau, and S. Das Sarma, Phys. Rev. Lett. 105, 077001 (2010).

[32] J. D. Sau, R. M. Lutchyn, S. Tewari, and S. Das Sarma, Phys. Rev. Lett. 104, 040502 (2010).

[33] H. Takayanagi, T. Akazaki, and J. Nitta, Phys. Rev. Lett. 75, 3533 (1995).

[34] T. Bauch, E. Hürfeld, V. M. Krasnov, P. Delsing, H. Takayanagi, and T. Akazaki, Phys. Rev. B 71, 174502 (2005).

[35] P. San-Jose, J. L. Lado, R. Aguado, F. Guinea, and J. Fernández-Rossier, Phys. Rev. X 5, 041042 (2015).

[36] J. Shabani, M. Kjaergaard, H. J. Suominen, Y. Kim, F. Nichele, K. Pakrouski, T. Stankevic, R. M. Lutchyn, P. Krogstrup, R. Feidenhans'1, S. Kraemer, C. Nayak, M. Troyer, C. M. Marcus, and C. J. Palmstrøm, Phys. Rev. B 93, 155402 (2016).

[37] M. Kjaergaard, F. Nichele, H. J. Suominen, M. P. Nowak, M. Wimmer, A. R. Akhmerov, J. A. Folk, K. Flensberg, J. Shabani, C. J. Palmstrøm, and C. M. Marcus, Nat. Commun. 7, 12841 (2016).

[38] M. Hell, M. Leijnse, and K. Flensberg, Phys. Rev. Lett. 118, 107701 (2017).

[39] F. Pientka, A. Keselman, E. Berg, A. Yacoby, A. Stern, and B. I. Halperin, Phys. Rev. X 7, 021032 (2017).

[40] M. Hell, K. Flensberg, and M. Leijnse, Phys. Rev. B 96, 035444 (2017).

[41] F. Nichele, A. C. C. Drachmann, A. M. Whiticar, E. C. T. O'Farrell, H. J. Suominen, A. Fornieri, T. Wang, G. C. Gardner, C. Thomas, A. T. Hatke, P. Krogstrup, M. J. Manfra, K. Flensberg, and C. M. Marcus, Phys. Rev. Lett. 119, 136803 (2017).

[42] H. Hoppe, U. Zülicke, and G. Schön, Phys. Rev. Lett. 84, 1804 (2000).

[43] X.-L. Qi, T. L. Hughes, and S.-C. Zhang, Phys. Rev. B 82, 184516 (2010).

[44] J. M. Byers and M.E. Flatté, Phys. Rev. Lett. 74, 306 (1995).

[45] S. G. den Hartog, C. M. A. Kapteyn, B. J. van Wees, T. M. Klapwijk, and G. Borghs, Phys. Rev. Lett. 77, 4954 (1996).

[46] G. Deutscher and D. Feinberg, Appl. Phys. Lett. 76, 487 (2000).

[47] A. L. Yeyati, F. S. Bergeret, A. Martin-Rodero, and T. M. Klapwijk, Nat. Phys. 3, 455 (2007).

[48] G.-H. Lee, K.-F. Huang, D. K. Efetov, D. S. Wei, S. Hart, T. Taniguchi, K. Watanabe, A. Yacoby, and P. Kim, Nat. Phys. 13, 693 (2017).

[49] We note that for $\nu=2$ [48] (or even fillings in general), pairs of Majoranas will be generated which will not be protected against hybridization into conventional fermions. Odd fillings, however, will always generate one protected unpaired Majorana zero mode.

[50] D. J. Clarke, J. Alicea, and K. Shtengel, Nat. Commun. 4, 1348 (2013).

[51] J. Klinovaja, A. Yacoby, and D. Loss, Phys. Rev. B 90, 155447 (2014). 
[52] J. Alicea and P. Fendley, Annu. Rev. Condens. Matter Phys. 7, 119 (2016).

[53] C. Reeg, J. Klinovaja, and D. Loss, Phys. Rev. B 96, 081301 (2017).

[54] T. Ojanen, Phys. Rev. B 87, 100506 (2013).

[55] C. Schrade, A. A. Zyuzin, J. Klinovaja, and D. Loss, Phys. Rev. Lett. 115, 237001 (2015).

[56] K. Flensberg, Phys. Rev. Lett. 106, 090503 (2011).

[57] J. L. Lado and J. Fernández-Rossier, Phys. Rev. B 90, 165429 (2014).

[58] F. Finocchiaro, F. Guinea, and P. San-Jose, 2D Mater. 4, 025027 (2017).

[59] See Supplemental Material at http://link.aps.org/ supplemental/10.1103/PhysRevLett.120.116801 for details on modeling, simulation parameters, analytical results, and additional Refs. [60-62].

[60] E. Prada and F. Sols, Eur. Phys. J. B 40, 379 (2004).

[61] J. Bardeen, R. Kümmel, A. E. Jacobs, and L. Tewordt, Phys. Rev. 187, 556 (1969).

[62] W. Kirk and P. Reed, Nanostructures and Mesoscopic Systems (Academic Press, 1992).

[63] S. B. Chung, X.-L. Qi, J. Maciejko, and S.-C. Zhang, Phys. Rev. B 83, 100512 (2011).

[64] J. Wang, Q. Zhou, B. Lian, and S.-C. Zhang, Phys. Rev. B 92, 064520 (2015).

[65] E. Prada, R. Aguado, and P. San-Jose, Phys. Rev. B 96, 085418 (2017). 